\title{
Surgeon Satisfaction During Endoscopic Management of Cerebrospinal Fluid Rhinorrhea: A Comparison Between Propofol-Remifentanil and Isoflurane-Remifentanil Anesthesia
}

\author{
Valliolah Hassani ${ }^{1}$, Mahzad Alimian ${ }^{1 *}$, Mohammad Farhadi ${ }^{1}$, Behrouz Zaman ${ }^{1}$, Masood \\ Mohseni ${ }^{1}$ \\ ${ }^{1}$ Department of Anesthesiology, Rasool Akram Hospital, Tehran University of Medical Sciences, Tehran, IR Iran
}

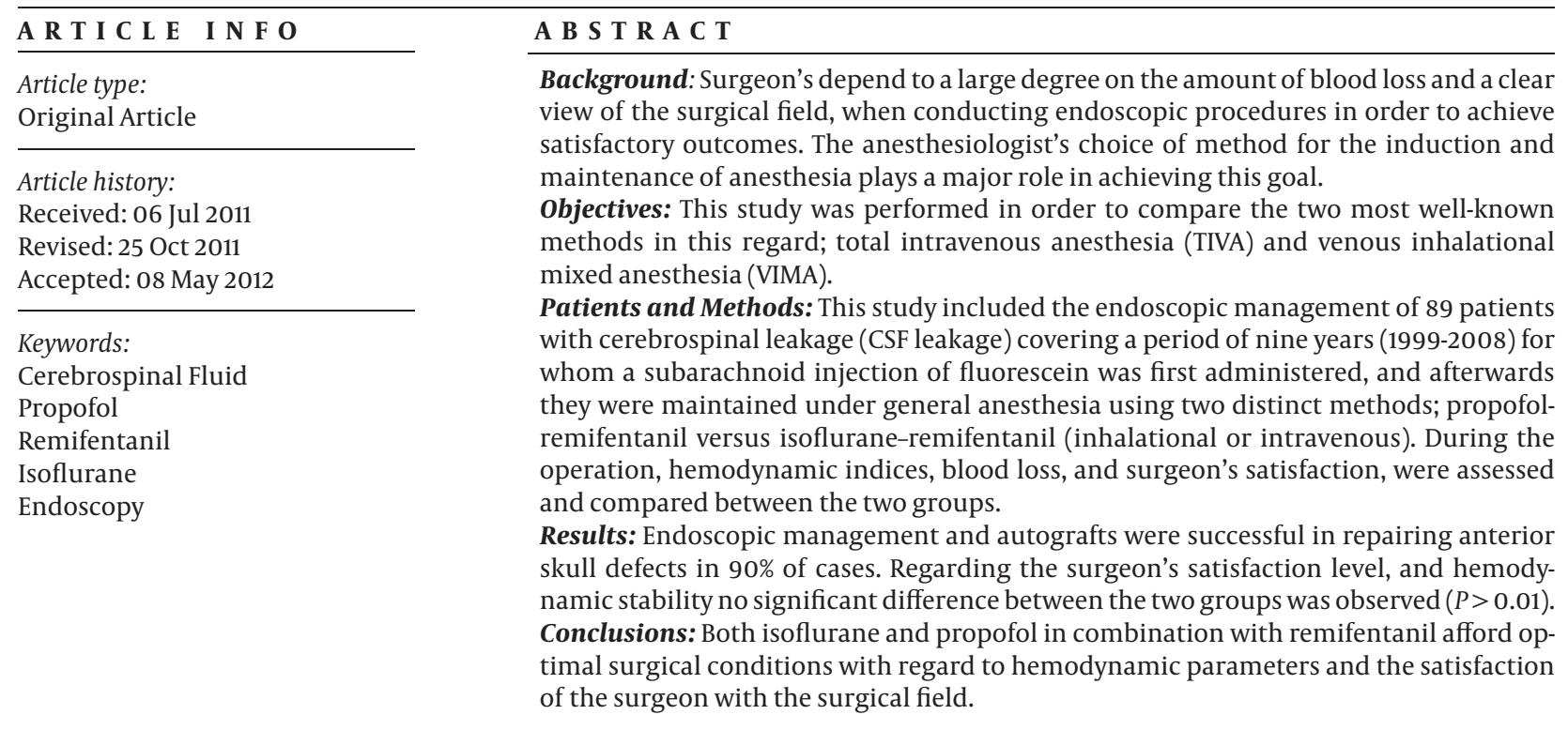

Implication for health policy/practice/research/medical education:

Surgeon satisfaction during endoscopy depends on the amount of blood loss and also the type of anesthesia that is applying for the surgery. Therefore, in this study two different types of surgery (TIVA and VIMA) are compared. We highly recommend to the surgeons and anesthesiologists to study this article.

Please cite this paper as:

Hasani V, Alimian M, Farhadi M, Zaman B, Mohseni M. Surgeon Satisfaction During Endoscopic Management of Cerebrospinal Fluid Rhinorrhea: A Comparison Between Propofol-Remifentanil and Isoflurane-Remifentanil Anesthesia. J Minim Invasive Surg Sci. 2012; 1(2): 62-6. DOI: 10.5812/jmiss.1839

\footnotetext{
* Corresponding author: Mahzad Alimian, Department of Anesthesiology, Rasool Akram Hospital, Tehran University of Medical Sciences, Tehran, IR Iran.Tel:+98-2164352326, Fax:+98-2166509059,E-mail: m-alimian@tums.ac.ir DOI:10.5812/jmiss.1839

Copyright (c) 2012, Minimally Invasive Surgery Research Center and Mediterranean \& Middle Eastern Endoscopic Surgery Association. This is an open-access article distributed under the terms of the Creative Commons Attribution License, which permits unrestricted use, distribution, and reproduction in any medium, provided the original work is properly cited.
}

\section{Background}

Anesthesiologists play a major role in the management of bleeding during micro-surgeries which require a clear, blood-less field for the surgeon to display his optimum expertise. This is demonstrated in particular by the way the anesthesiologist chooses to maintain anesthesia, whether inhalational or intravenous, in order to gain 
the surgeon's approval as well as providing the patient with the least hemodynamic changes, intra-operative bleeding, and a shorter recovery time. Cerebrospinal fluid leakage from anterior skull base defects is one of the remaining challenges of endoscopic surgery in recent years (1) (Figure 1). These leaks can be primary (spontaneous) or secondary (due to head trauma). In most cases endoscopic nasal surgery for CSF leak repair has almost completely replaced more traumatic transcranial and extracranial procedures (2). However, more investigation is still needed with a greater focus on the method chosen for anesthesia in order to propose that this micro-surgical method is a safe and effective one for the repair of skull base defects.

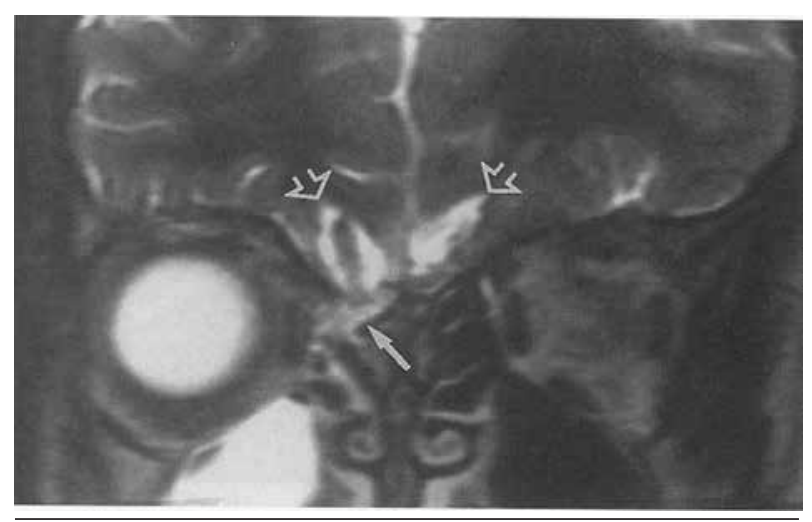

Figure 1. CSF-Leakage From Anterior Skull Base (MRI T12)

\section{Objectives}

In this study we have reviewed two distinct methods for anesthesia during the endoscopic management of patients with CSF rhinorrhea; intravenous and inhalational.

\section{Patients and Methods}

This cohort study includes the endoscopic repair of CSF leakage in 89 patients during a nine year period (19992008), in the Rasool Akram Hospital, Tehran, Iran. The patients under study were elected through simple non-random convenience sampling. Patients were consecutively enrolled based on the previously mentioned surgical inclusion criteria. Among these cases, 48 had traumatic etiology, 27 were iatrogenic, and the rest (14 cases) had spontaneous CSF leakage. Fine cut axial and coronal computed tomography (CT) scans were obtained from all patients before surgery. Also, magnetic resonance imaging (MRI) and CT-cisternography with contrast were used for some patients.

According to the surgeon's policy and decision before the induction of anesthesia, an intrathecal injection of fluorescein was performed (Figure 2). Approximately 9.5 cc of CSF was mixed with 0.25 cc of a $10 \%$ fluorescein solution, slowly injected into the subarachnoid space through a $22 \mathrm{G}$ French spinal needle in the L3-L4 inter-

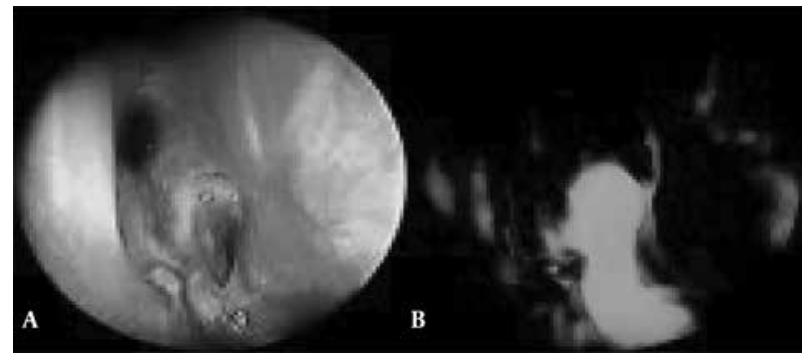

Figure 2. A) CSF-Leakage With Intrathecal Fluorescein, B) Dark Field

space. The patients were held in a slight Trendelenburg position (15-30 degrees) for 30 minutes. Based on the study protocol, none of the patients received preoperative or intraoperative local vasoconstrictors. The patients were premedicated with midazolam $0.05 \mathrm{mg} / \mathrm{kg}$ and fentanyl $3 \mu \mathrm{g} / \mathrm{kg}$. Induction of anesthesia was performed with $5 \mathrm{mg} / \mathrm{kg}$ sodium thiopental and $0.5 \mathrm{mg} / \mathrm{kg}$ atracurium as the muscle relaxant. Intubation was performed after mask ventilation with $100 \%$ oxygen, and patients were allocated to receive either intravenous or inhalational anesthesia. The first group $(\mathrm{n}=52)$ received $100 \mu \mathrm{g} / \mathrm{kg} / \mathrm{min}$ propofol and $0.2 \mu \mathrm{g} / \mathrm{kg} / \mathrm{min}$ remifentanil infusion for maintenance of anesthesia and the second group $(n=37)$ were maintained under an inhalational technique with $1.5 \%$ isoflurane combined with remifentanil infusion (0.2 $\mu \mathrm{g} / \mathrm{kg} / \mathrm{min})$.

Preoperatively, patients were examined with an endoscope and, if necessary, with a blue light and a dark field (Figure 3). The sites of examination for CSF leak-

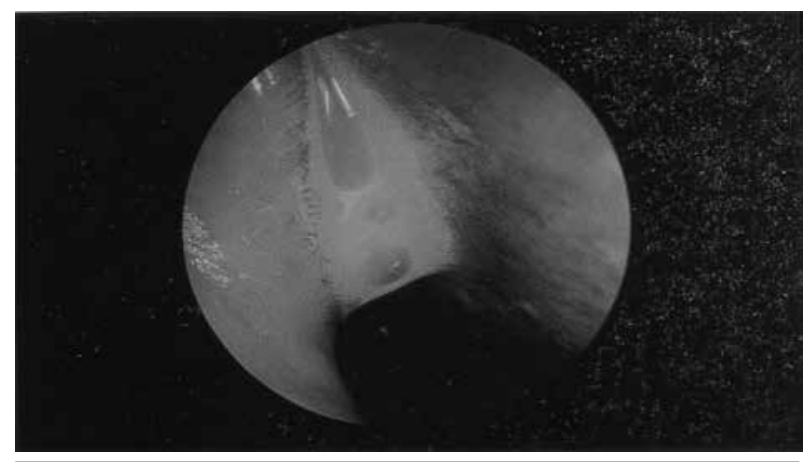

Figure 3. Blue light

age were the Eustachian tube, cribriform plate, middle meatus, and the ostium of the sphenoid. The surgical approach was made through a standard functional endoscopic sinus surgery (FESS) technique. Complete ethmoidectomy was initially carried out to enable surgeons to precisely identify the location of the CSF leak. In cases with a defect of approximately $5 \mathrm{~mm}$ or larger, the otolaryngologists attempted to use a piece of cartilage or bone as a support underlay at the edges of the bony defect area (Figure 4). The cartilage or bone grafts were harvested either from the septum or from the lower or middle turbinate. Nasal packing was also used to support the graft and this consisted of surgi- 


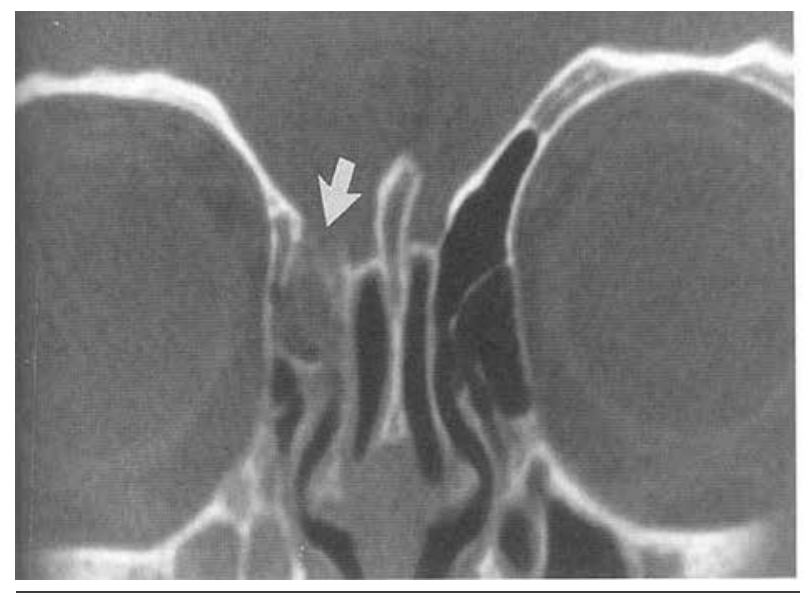

Figure 4. CT Defect in Fovea Ethmoidalis

cel, gelfoam and fibrin glue. At the end of the surgery, the surgical field was graded in terms of bleeding by the surgeon using the scale developed by Boezaart and van der Merwe in 1995 (3) (Sidebar):A Boezaart score of less than 4 was considered as surgeon's satisfaction. Age and weight of patients as well as hemodynamic parameters including; systolic blood pressure and heart rate, were recorded before and every 15 minutes during the surgery. The important factors from our point of view include; the amount of bleeding, hemodynamic indices and the duration of the surgery, which were all evaluated. Data are presented as mean (standard deviation) and frequency (percentage), as appropriate. Baseline characteristics of the two groups were analyzed with a Student's t-test for continuous data and a Chi-square test for categorical analysis. All of the comparisons were two tailed and $P<0.05$ was considered statistically significant. Statistical analyses were performed with SPSS version 9.0 software (SPSS Inc. Chicago, IL).

\section{Results}

Demographic variables and hemodynamic profile of patients are presented in Table. No significant difference in demographic indices, as well as blood pressure and heart rate, were observed between the two groups. The cause of CSF rhinorrhea was determined to be traumatic (53.8\%), spontaneous (14.6\%), and iatrogenic (31.6\%). The trend of mean blood pressure and heart rate were not significantly different between the two groups in 15 minute interval assessments (data not shown). Controlled hypotension, other than drugs for the maintenance of anesthesia, was not intended for the two groups and the hemodynamic profile of pa-

\begin{tabular}{ll}
\hline Sidebar. Boezaart Grading Scale for Scoring of Surgical Field Bleeding \\
\hline & Assessment \\
\hline 0 & No bleeding (cadaveric conditions) \\
1 & Slight bleeding-no suctioning required \\
2 & Slight bleeding-occasional suctioning required \\
3 & Slight bleeding-frequent suctioning required. Bleeding threatens surgical field a few seconds after suction is removed. \\
4 & Moderate bleeding-frequent suctioning required. Bleeding threatens surgical field directly after suction is removed. \\
5 & $\begin{array}{l}\text { Severe bleeding-constant suctioning required. Bleeding appears faster than can be removed by suction; surgical field severely } \\
\text { threatened and surgery usually not possible. }\end{array}$ \\
\hline
\end{tabular}

\begin{tabular}{|c|c|c|c|}
\hline & Group I (TIVA) & Group II (VIMA) & Pvalue \\
\hline \multicolumn{4}{|c|}{ Demographic and Pre-induction Characteristics } \\
\hline Age, y, mean \pm SD & $47.4 \pm 23.2$ & $48.3 \pm 25.8$ & NS \\
\hline Weight, $\mathrm{kg}$, mean \pm SD & $64.3 \pm 4.7$ & $63.8 \pm 7.8$ & NS \\
\hline Systolic blood pressure, $\mathrm{mmHg}$, mean \pm SD & $123.2 \pm 5.2$ & $116.8 \pm 4.3$ & NS \\
\hline Heart rate, beat $/ \mathrm{min}$, mean \pm SD & $93.7 \pm 12.5$ & $84.5 \pm 10.4$ & NS \\
\hline \multicolumn{4}{|c|}{ Hemodynamic Parameters and Outcome of Operation } \\
\hline Systolic blood pressure, $\mathrm{mmHg}$, mean \pm SD & $108.4 \pm 14.5$ & $106.8 \pm 11.7$ & NS \\
\hline Diastolic blood pressure, $\mathrm{mmHg}$, mean $\pm \mathrm{SD}$ & $65.1 \pm 2.2$ & $64.3 \pm 1.8$ & NS \\
\hline Heart rate, beat/min, mean \pm SD & $78.3 \pm 4.8$ & $81.4 \pm 13.2$ & NS \\
\hline Bleeding, ml, mean \pm SD & $351.4 \pm 70.4$ & $185.4 \pm 83.6$ & $<0.001$ \\
\hline Time of surgery, min, mean \pm SD & $240.7 \pm 25.4$ & $235.7 \pm 18.4$ & NS \\
\hline Surgeon's satisfaction, No. (\%) & $51(98.2)$ & $36(97.3)$ & NS \\
\hline Time of recovery, min, mean \pm SD & $23.4 \pm 2.7$ & $26.7 \pm 8.5$ & NS \\
\hline
\end{tabular}

Abbreviations: NS, not significant; TIVA, total intravenous; VIMA, venous inhalational mixed anesthesia 
tients before awakening was not statistically different between the two groups (Table). The duration of the surgery as well as the time taken for full recovery was similar between the two groups. The amount of bleeding in the total intravenous anesthesia (TIVA) group was greater than the blood loss in the venous inhalational mixed anesthesia (VIMA) group, however, the percentage of a favorable quality of surgical field (score $<4$ ) measured as surgeon satisfaction was not statistically different between the two groups (Table). Regarding the surgical repairs, only 5 failures occurred, 4 of which required a craniotomy and the one remaining was repaired after a second endoscopic procedure. Approximately $90.76 \%$ of cases were successfully treated with $84.6 \%$ effectively repaired on the first attempt.

\section{Discussion}

Since 1926 when Dandy described the first case using a bifrontal craniotomy, this approach remained the main stem surgical treatment of choice until the Dohlman study reported the first treatment with an extracranial nonendoscopic approach (4). Wigand, in 1981, was the first to describe the use of an endoscope in the treatment of CSF rhinorrhea. Also, utilizing fluorescein facilitated detection of the leakage site, being relatively safe without any significant complications $(5,6)$. In our study, the most common site for CSF leak was the cribriform plate, which is well known as being the thinnest part of the skull base, in spontaneous and post-op cases. Several medications and techniques of anesthesia have been tried to optimize the quality of the surgical field in endoscopic surgery (711). In this research, as previously mentioned, we selected the two most common methods of anesthesia utilized in various circumstances; TIVA and VIMA. For some years, isoflurane and propofol have been shown to provide optimal hemodynamic and surgical conditions for gross and micro-surgeries. These drugs, especially in combination with remifentanil, decrease blood pressure as they have an effect on myocardial contractility and systemic vascular resistance, bleeding decreases which leads to the surgeon's increased level of satisfaction, at the same time it also reduces patient morbidity. As a result of their context-sensitive half-time, these drugs are rapidly eliminated with a consequent shortened post-operative recovery period (12). No major complications resulted during operations which selected TIVA or VIMA anesthetic approaches, as demonstrated in our study. The total blood loss in the two groups was not very great, however, even a small amount of bleeding in this type of microsurgery can interfere with an optimal operation. Isoflurane by decreasing systemic vascular resistance may provide better control of bleeding than propofol, but the collected data shows no significant difference in blood pressure and surgeon satisfaction levels. In this study the amount of blood loss was estimated and we used a scale to evalu- ate the satisfaction of the surgical team. This measurement tool is appropriate considering that the direct objective of the anesthesia in microsurgery is to create a clean surgical field rather than to reduce blood loss. Recently, a multi-center standardized reliability analysis has verified the inter- and intra-observer reliability of the Boezaart scale (13). Thus far, no other study has been reported that compares these two techniques of anesthesia in CSF leakage repair, however, we were able to provide a favorable level of surgeon's satisfaction regarding the blood-free surgical field and the amount of bleeding. As a result of this complication-free intra-operative period, we were able to provide the patients with a safe, short and relatively pleasant postoperative recovery stay. This investigation could be the starting point for more research in this field, and specifically in endoscopic procedures. In order to achieve the best results attention to; surgeon's satisfaction, hemodynamic stability, blood loss, recovery period, and last but most importantly, patients' safety with the least morbidity and mortality should be included in further studies.

\section{Acknowledgments}

None declared.

\section{Authors' Contribution}

None declared.

\section{Financial Disclosure}

None declared.

\section{Funding/Support}

None declared.

\section{References}

1. Snyderman CH, Kassam AB, Carrau R, Mintz A. Endoscopic Reconstruction of Cranial Base Defects following Endonasal Skull Base Surgery. Skull Base. 2007;17(1):73-8.

2. Lopatin AS, Kapitanov DN, Potapov AA. Endonasal endoscopic repair of spontaneous cerebrospinal fluid leaks. Arch Otolaryngol Head Neck Surg. 2003;129(8):859-63.

3. Boezaart AP, van der Merwe J, Coetzee A. Comparison of sodium nitroprusside- and esmolol-induced controlled hypotension for functional endoscopic sinus surgery. Can J Anaesth. 1995;42(5 Pt 1):373-6.

4. Schlosser RJ, Bolger WE. Endoscopic management of cerebrospinal fluid rhinorrhea. Otolaryngol Clin North Am. 2006;39(3):523-38, ix.

5. Nemergut EC, Dumont AS, Barry UT, Laws ER. Perioperative management of patients undergoing transsphenoidal pituitary surgery. Anesth Analg. 2005;101(4):1170-81.

6. Wax MK, Ramadan HH, Ortiz O, Wetmore SJ. Contemporary management of cerebrospinal fluid rhinorrhea. Otolaryngol Head Neck Surg. 1997;116(4):442-9.

7. Alimian M, Mohseni M. The effect of intravenous tranexamic acid on blood loss and surgical field quality during endoscopic sinus surgery: a placebo-controlled clinical trial. J Clin Anesth. 2011;23(8):611-5.

8. Mohseni M, Ebneshahidi A. The effect of oral clonidine premedi- 
cation on blood loss and the quality of the surgical field during endoscopic sinus surgery: a placebo-controlled clinical trial. Anesth. 2011;25(4):614-7.

9. Riegle EV, Gunter JB, Lusk RP, Muntz HR, Weiss KL. Comparison of vasoconstrictors for functional endoscopic sinus surgery in children. Laryngoscope. 1992;102(7):820-3.

10. Shaw CL, Dymock RB, Cowin A, Wormald P. Effect of packing on nasal mucosa of sheep.J Laryngol Otol. 2000;114(7):506-9.

11. Wormald PJ. The surgical field in endoscopic sinus surgery. In: Wormald PJ, editor. Endoscopic Sinus Surgery-Anatomy, Three-
Dimensional Reconstruction, and Surgical Technique. New York: Thieme; 2008. p. 7-12.

12. Lindstrom DR, Toohill RJ, Loehrl TA, Smith TL. Management of cerebrospinal fluid rhinorrhea: the Medical College of Wisconsin experience. Laryngoscope. 2004;114(6):969-74.

13. Athanasiadis T, Beule A, Embate J, Steinmeier E, Field J, Wormald PJ. Standardized video-endoscopy and surgical field grading scale for endoscopic sinus surgery: a multi-centre study. Laryngoscope. 2008;118(2):314-9. 